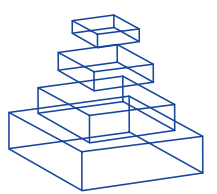

\title{
Layer, column and cell-type specific genetic manipulation in mouse barrel cortex
}

\author{
Rachel Aronoff ${ }^{*}$ and Carl C.H. Petersen ${ }^{*}$, \\ * Laboratory of Sensory Processing, Brain Mind Institute, Ecole Polytechnique Fédérale de Lausanne, \\ Lausanne, Switzerland.
}

Sensory information is processed in distributed neuronal networks connected by intricate synaptic circuits. Studies of the rodent brain can provide insight into synaptic mechanisms of sensory perception and associative learning. In particular, the mouse whisker sensorimotor system has recently begun to be investigated through combinations of imaging and electrophysiology, providing data correlating neural activity with behaviour. In order to go beyond such correlative studies and to pinpoint the contributions of individual genes to brain function, it is critical to make highly controlled and specific manipulations. Here, we review recent progress towards genetic manipulation of targeted genes in specific neuronal cell types located in a selected cortical layer of a well-defined cortical column of mouse barrel cortex. The unprecedented precision of such genetic manipulation within highly specific neural circuits may contribute significantly to progress in understanding the molecular and synaptic determinants of simple forms of sensory perception and associative learning.

Keywords: barrel cortex, lentivirus, genetic manipulation, NMDA receptors

\section{ORGANIZATION OF THE NEOCORTEX}

Sensory information is processed in the neocortex by highly ordered neuronal networks. Tangential to the cortical surface, representations of the sensory periphery are organized into well-ordered maps. The most intensely studied example is the primary visual cortex, which is arranged with superimposed maps of retinotopy, ocular dominance and orientation (Bonhoeffer and Grinvald, 1991). Other prominent cortical maps are the tonotopic organization of auditory cortex (Kalatsky et al., 2005), taste maps in gustatory cortex (Accolla et al., 2007) and somatotopic maps in primary somatosensory cortex (Kaas, 1991). Mapping functionally related sensory information onto nearby cortical regions is thought to minimize axonal wiring length and simplify the synaptic circuits underlying correlationbased associational plasticity.
Another form of cortical organization is clearly evident orthogonal to these tangentially distributed maps. Normal to the cortical surface, the neocortex can be divided into distinct layers containing various classes of neurons, each contributing differently to cortical computation (Krupa et al., 2004). In primary sensory cortices, information from the periphery arrives primarily from the thalamic innervation of layer 4 , also known as the granular cell layer. Cortical layers 1, 2 and 3 form the superficial layers, also known as the supragranular layers, which are likely the most integrative layers, gathering sensory information and distributing output to other cortical areas. The deep layers 5 and 6 form the output layers of the neocortex, innervating both subcortical and cortical areas. Anatomical and electrophysiological studies have begun to shed light on the synaptic architecture of cortical microcircuits 
(Binzegger et al., 2004; Silberberg et al., 2005; Thomson and Lamy, 2007). The next obvious step is to relate the wiring diagram of these neuronal networks to their functional operation. This is a difficult challenge, but the mouse barrel cortex offers promising avenues for future quantitative research.

\section{ORGANIZATION OF THE MOUSE BARREL CORTEX}

Rodents are nocturnal, living in tunnels. The extensive array of mystacial vibrissae under active motor control serves as a sensitive tactile organ, which can provide information relating to the structure of the immediate surroundings (Kleinfeld et al., 2006; Petersen, 2007). A surprisingly large part of the mouse brain is concerned with processing sensorimotor information relating to these whiskers on the snout (Figure 1A). A remarkably high degree of organization is obvious in the primary somatosensory cortex, in which a clear pattern of cytoarchitectonic units termed 'barrels' are observed in perfect match with the arrangement of the whiskers on the snout of the mouse (Woolsey and Van der Loos, 1970) (Figure 1B). These somatotopically arranged barrels are found exclusively in neocortical layer 4 (Figure 1C), and are likely formed during development through the patterned innervation of thalamocortical axons from the ventral posterior medial nucleus. Much of the cortical circuitry is arranged in a columnar fashion extending above and below the layer 4 barrel, and this defines a cortical barrel column (Petersen and Sakmann, 2001; Shepherd et al., 2003). Information relating to deflection of an individual whisker is processed primarily within the homologous barrel column. However, there is also clear evidence for important lateral interactions, as found through examination of long-range axonal projections in supragranular and infragranular layers and through the broad subthreshold receptive fields (Brecht, 2007; Petersen, 2007).

In order to simplify experimental data and its interpretation, many researchers have found it useful to concentrate on the sensory information available from just a single whisker, by trimming all other whiskers. Under these conditions it is possible to quantify the detailed kinetics of whisker movements and the precise moment when an individual whisker touches an object and correlate this information with cortical sensory processing (Crochet and Petersen, 2006; Ferezou et al., 2006, 2007). Rodents with a single whisker can perform a number of whiskerdependent tasks, the best known of which is the gap-crossing task (Hutson and Masterton, 1986). In this behaviour, the rodent is placed on one elevated platform from which it has to reach across and jump to a target platform where a reward is located. The rodents are forced to use their whisker to locate the target platform by performing the experiments in darkness and making the gap so large that the nose and paw cannot reach across. This behaviour depends upon the operation of the barrel cortex (Hutson and Masterton, 1986). Further experimental evidence suggests that at least part of the associational learning of this single whisker mediated behaviour may take place within the homologous cortical column (Harris et al., 1999).

Because defined barrel columns can be reliably identified between animals, it seems likely that a great deal can be learned from detailed investigation of the synaptic circuits underlying a single cortical barrel column. However, this information must be integrated with data relating to synaptic interactions with other cortical and subcortical brain areas and
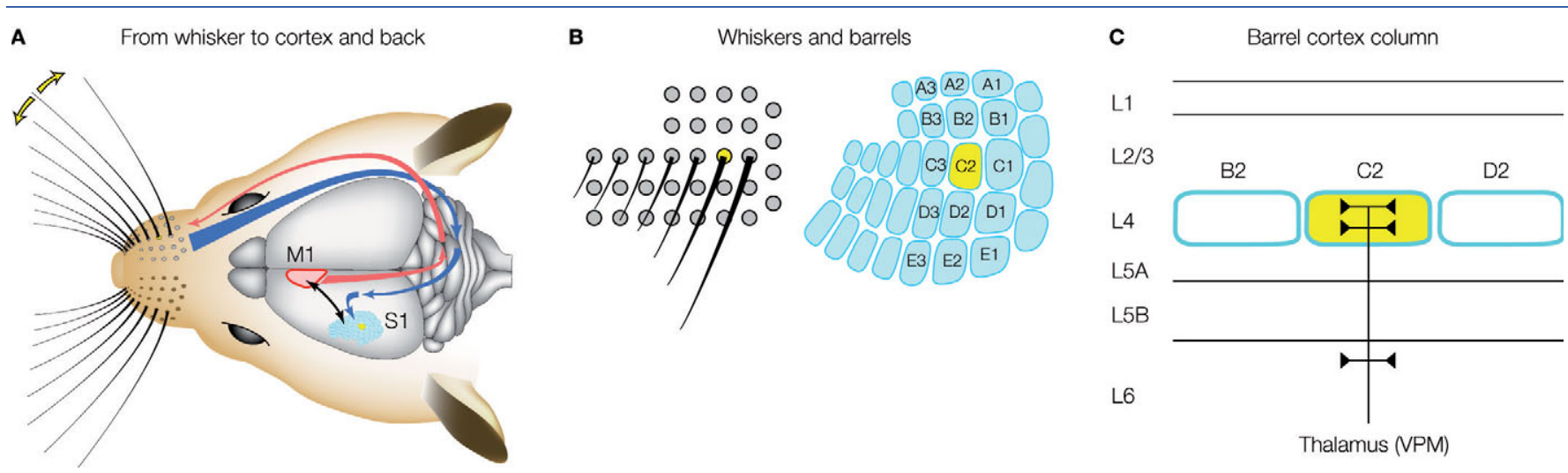

Figure 1 I The barrel cortex. (A) An impressive array of whiskers on the snout of the rodent sends sensory information to the primary somatosensory barrel cortex (S1) via the brainstem and the thalamus. The barrel cortex signals to motor cortex (M1), which in turn regulates whisker movements. Active processing of tactile whisker information therefore appears to be an important feature of this sensory pathway. (B) The layout of the whiskers (left) is precisely matched by the layout of the barrels (right) in primary somatosensory cortex. Mice and rats have the same layout of whiskers and a standard nomenclature has been developed. The $\mathrm{C} 2$ whisker and barrel are highlighted in yellow. (C) A barrel column is arranged in different layers. Single whisker sensory information from ventral posterior medial (VPM) thalamus arrives predominantly in a single layer 4 barrel. The supragranular layer $2 / 3$ and the infragranular layers $5 / 6$ are thought to perform integrative functions. Modified and reproduced with permission from Petersen (2007). Copyright from Neuron, Cell Press. 
with data about the physiological activity of the underlying neuronal networks during learning and behaviour. The growing body of quantitative data relating synaptic circuits in the barrel cortex to functional activation (Sarid et al., 2007) may thus lead to significant progress in our understanding of the synaptic mechanisms underlying sensory perception.

\section{LAYER, COLUMN AND CELL-TYPE SPECIFIC GENETIC MANIPULATION OF THE MOUSE BARREL CORTEX}

In order to fully exploit the barrel cortex as a model system for active sensory processing, we need to be able to make precise manipulations in neurons of a defined barrel column. Recent advances in genetic manipulations of mice provide increasing levels of sophistication in spatiotemporal regulation of gene expression in specific cells localized to defined regions of the brain. The genetic identification of molecular pathways underlying different aspects of cortical functional organization is also likely to lead to new therapeutic targets.

Since the first genetic studies of the barrelless mutation (Welker et al., 1996), many further genes have been identified as critical for barrel cortex formation. However, genetic lesions often affect many brain areas since the gene products are widely expressed and may also alter brain development. Under such conditions, it is difficult to attribute specific functions to individual genes. The development of systems for spatiotemporally controlled knockout of genes has therefore been crucial in the field of molecular neurobiology.

The first breakthrough, developed by the Tonegawa laboratory, came with the application of the Cre-loxP system to the brain. In their landmark papers, Tsien et al. (1996a,b) described a method to specifically remove NMDA receptors from the CA1 region of the hippocampus, while leaving NMDA receptors functional in all other brain regions. This was achieved by combining two genetically engineered mice. One mouse line was generated by gene targeting strategies to insert loxP sites surrounding the second half of the NMDA receptor 1 (NR1) gene, making a so-called floxed (flanked by loxP sites) NR1 gene. Expression and function of the NR1 gene, which encodes an essential subunit required for all NMDA receptors, was unaffected by these targeted insertions. The second transgenic mouse expressed Cre recombinase as a transgene under the $\alpha$ CaMKII promoter. In a number of independent mouse lines they found that functional Cre was only expressed in the CA1 pyramidal neurons. By interbreeding these two genetically engineered mice, the NR1 gene was knocked out specifically in the CA1 neurons, by the activity of Cre upon the loxP sites, and thus NMDA receptors were also eliminated. This Cre-loxP strategy has been found to be robust and generally applicable. However, it critically relies upon expressing Cre under control of cell-type specific promoters. Although a number of promoters have been identified and shown to drive Cre in stereotypical and defined patterns, it seems unlikely that this will be sufficient to target specific cortical columns in the mammalian brain. For example, the current most specific knockout of NMDA receptors in the neocortex removes the NR1 gene from the entire neocortex during development (Iwasato et al., 2000).

Gene transduction using viral vectors forms a promising strategy for localized genetic manipulation of the neocortex. Non-replicative lentiviral vectors (Deglon et al., 2000; Naldini et al., 1996) have been shown to offer long-term stable gene expression in the neocortex (Aronoff and Petersen, 2006, 2007; Dittgen et al., 2004; Komai et al., 2006). Lentivirus can be directly injected into mouse barrel cortex using very fine glass micropipettes (Figure 2A), and
A Lentivector injection into barrel cortex

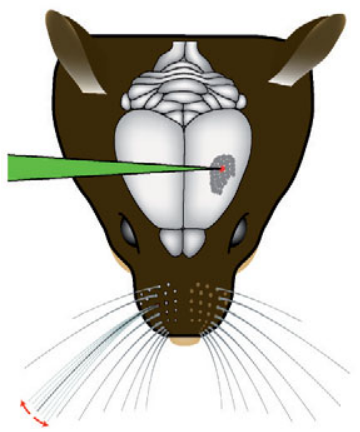

B Lentivector-mediated expression of GFP in L2/3 pyramidal neurons

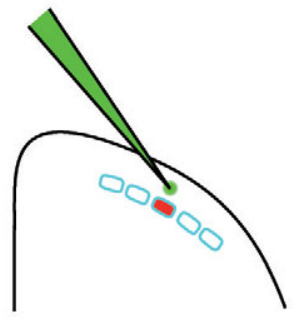

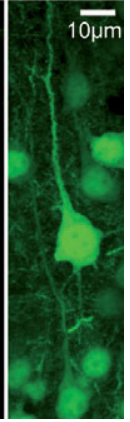

Figure 2 I Layer and cell-type specific genetic manipulation of the barrel cortex. (A) Lentivirus can be injected directly into the mouse barrel cortex using very fine glass micropipettes. (B) Lentivector injections result in highly localized gene transduction, limited to $\sim 200 \mu \mathrm{m}$ around the injection site. Injection of GFP-expressing lentivector into layer 2/3, therefore results in highly specific GFP expression in layer 2/3 and not elsewhere (left). GFP expression in pyramidal neurons is driven by the $\alpha$ CaMKIl promoter (right). Modified and reproduced with permission from Aronoff and Petersen (2007). Frontiers in Integrative Neuroscience. 
the injection process itself causes little damage. The viral particles do not diffuse far from the injection site, and typically we find a local transduction of neurons within a diameter of $\sim 200 \mu \mathrm{m}$. Transgene expression can be directly visualized by including the coding sequence for green fluorescent protein (GFP) in the transfer vector (Figure 2B). Pseudotyping with

A Intrinsic optical imaging

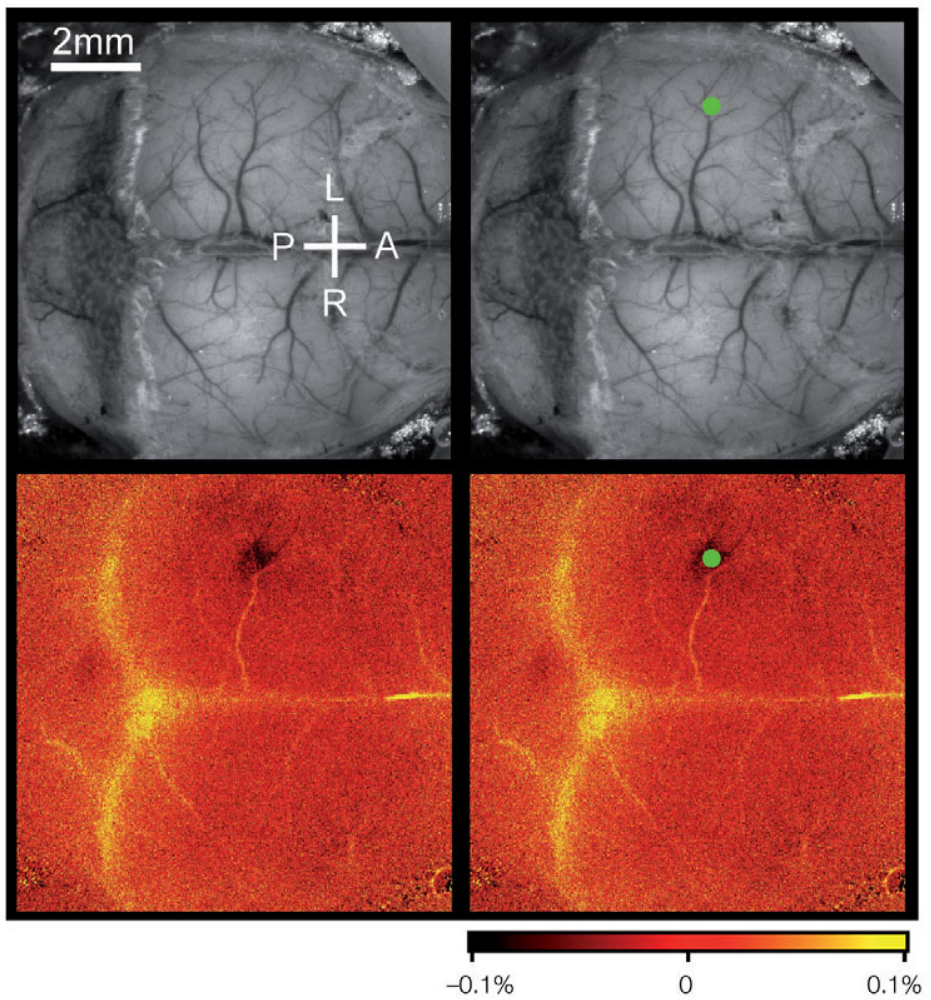

B C2-targeted lentivector injection
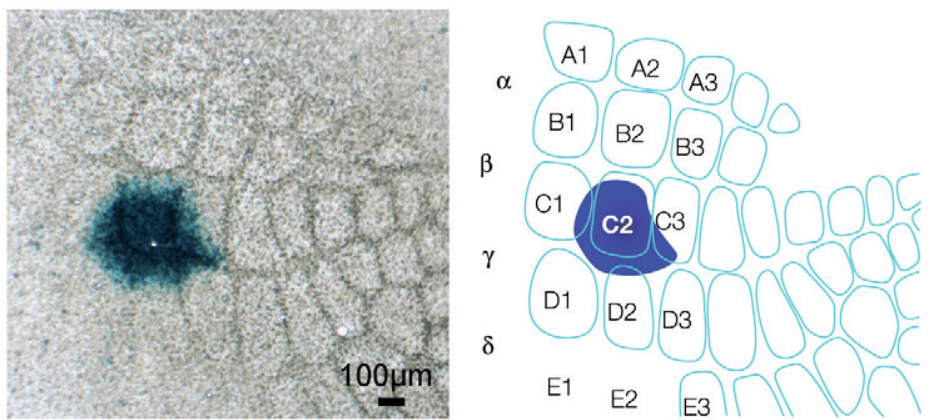

Figure 3 | Column specific genetic manipulation of the barrel cortex. $(\boldsymbol{A})$ Intrinsic optical imaging through the intact mouse skull can be used to non-invasively map the neocortex. Deflection of the C2 whisker evokes a localized hemodynamic signal highlighted by a green dot. The functional mapping can be related to the blood vessel layout allowing targeted craniotomy and lentivector injection. $(\boldsymbol{B})$ Injection of lentivector was targeted to the $\mathrm{C} 2$ barrel column through intrinsic optical imaging. The lentivector expressed Cre recombinase and was injected into a ROSA26R Cre-reporter mouse, which expresses LacZ in the presence of Creactivity. Modified and reproduced with permission from Aronoff and Petersen (2007). Frontiers in Integrative Neuroscience. vesicular stomatitis viral glycoprotein allows entry into neurons by vector particles, but neurons remote from the injection site are not transduced. Injection of lentivector into layer $2 / 3$ can result in transgene expression specifically within layer $2 / 3$ (Figure $2 B$, left). Lentivectors therefore provide convenient tools for layer-specific genetic manipulation in the mouse barrel cortex.

First order cell-type specificity can be encoded through the promoter driving transgene expression in the lentivector. In the neocortex, $\alpha \mathrm{CaMKII}$ is specifically expressed in the excitatory glutamatergic neurons. Using a 1.3-kb fragment of the $\alpha$ CaMKII promoter in lentivectors results in selective transgene expression in pyramidal neurons (Figure 2B, right) (Aronoff and Petersen, 2007; Dittgen et al., 2004), with only a very small fraction of expressing cells being GABAergic neurons. In future studies, it will be of great interest to examine whether further specificity for different types of pyramidal neurons and other cell-types in the neocortex can be engineered into lentivectors using other promoters or combinatorial genetic strategies.

The lateral dimensions of the cluster of transduced neurons following a lentivector injection into the neocortex are on the order of $\sim 200 \mu \mathrm{m}$, similar to the width of individual mouse cortical barrel columns. It would therefore seem likely that one could target a specific single cortical column for genetic manipulation. However, the somatotopic map of the barrel cortex is not directly visible in the living animal. It is therefore necessary to functionally map the cortex. Intrinsic optical imaging can be carried out through the intact skull of mice, revealing the functional location of the representation of a single whisker. In our experiments, we typically focus on the $\mathrm{C} 2$ whisker and its associated C2 barrel column (Figure 3A). Lentivector injections can subsequently be targeted to this specific cortical column. Expression of Cre can be functionally tested by injecting into mice carrying a $\mathrm{LacZ}$ reporter gene, which is conditionally expressed following Cre-mediated excision of a floxed STOP cassette (Soriano, 1999). Injecting Cre-expressing lentivector into the $\mathrm{C} 2$ column of such a reporter mouse after mapping by intrinsic optical imaging results in labelling predominantly of the $\mathrm{C} 2$ barrel column (Figure 3B). Targeted injection of lentivector expressing GFP and Cre can therefore be applied to make layer, cell-type and column specific genetic manipulations.

\section{AXONAL TRACING USING LENTIVECTORS}

The highly specific expression of GFP mediated by lentivectors in itself has proven useful in analysing axonal output patterns from a given layer and column of the barrel cortex. Broser et al. (2007) 
used lentivirus to study the effects of sensory deprivation by whisker trimming upon excitatory axonal arbors within barrel cortex. Layer $2 / 3$ pyramidal neurons located in spared cortex bordering upon deprived cortex were found to project axons asymmetrically, favouring the neighbouring region of spared cortex. Long-range axonal projections from the barrel cortex have also been studied and provided evidence for a prominent innervation of whisker motor cortex (Ferezou et al., 2007) (Figure 4). Such a sensorimotor loop from barrel cortex to motor cortex could be intimately involved in active sensory perception. Lentivector-labelled axons from cells with soma in the $\mathrm{C} 2$ barrel column course through the lower layers of the neocortex (Figure 4A) and give rise to a columnar innervation of motor cortex which spreads laterally in layer 1 (Figure 4B). In turn, lentivector injections in whisker motor cortex have shown that pyramidal neurons project directly onto motor neurons of the facial nucleus, as well as into the brainstem reticular formation (Grinevich et al., 2005). These anatomical tracing studies complement results from older methods lacking genetic specificity in which it can be difficult to separate retrograde and anterograde labelling.

\section{HIGHLY SPECIFIC KNOCKOUT OF NMDA RECEPTORS IN THE MOUSE BARREL CORTEX}

Although lentivectors can be used to direct transgene expression in layer, column and cell-type specific manner, they cannot be used directly to knockout a target gene. The lentivirus is a retrovirus, so its RNA is reverse transcribed in infected cells and subsequently the DNA is integrated into an unspecified location of the host genome. In order to knockout a specific gene, it is therefore necessary to use a two component system. In our study, we combined lentivector expressing Cre with gene-targeted mice carrying a floxed NMDA receptor 1 gene (Aronoff and Petersen, 2007). Transduced cells were visualized through co-expression of GFP from the same lentivector (Figure 5A). Using this manipulation, the floxed NR1 gene could be deleted in a layer, column and cell-type specific manner, since the genetic knockout only occurs in GFP/Cre-expressing neurons. All surrounding uninfected neurons are phenotypically wild-type (Figure 5B). Electrophysiological analysis of floxed NR1 mice revealed that longlasting NMDA receptor-dependent currents at positive potentials were strongly reduced or absent in cells expressing GFP and Cre (Figure 5C). NMDAreceptor dependent long-term potentiation was also found to be reduced in these Cre-expressing neurons of floxed NR1 mice (Aronoff and Petersen, 2007). Such layer- and cell-type- specific knockout
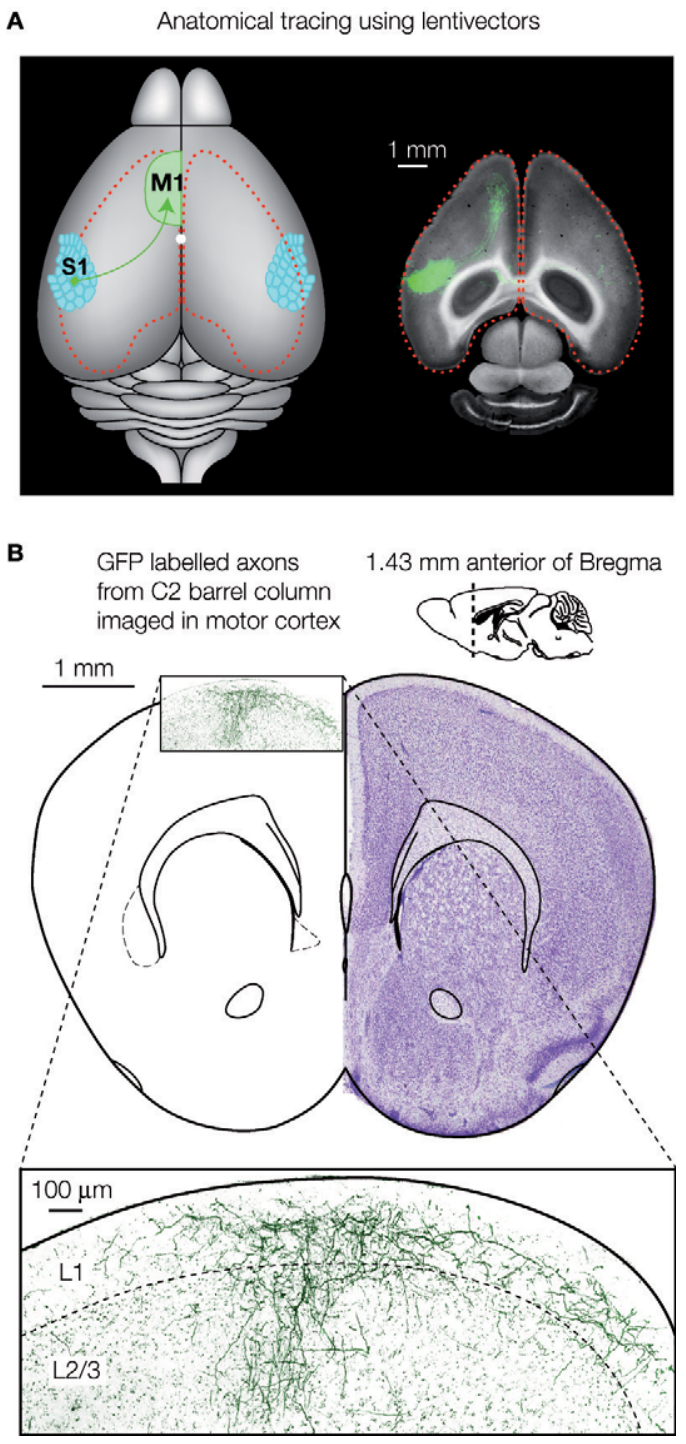

Figure 4 | Axonal tracing using lentivectors. (A) Targeted injection of GFP expressing lentivectors into the mouse C2 barrel column, allows the analysis of the long-range axonal output. A prominent innervation of motor cortex is visualized in horizontal brain slices. (B) GFP labelled axons originating from pyramidal neurons in the $\mathrm{C} 2$ barrel column are shown in a coronal section of motor cortex ( 1.4 mm anterior of Bregma). Panel A modified and reproduced with permission from Ferezou et al. (2007). Copyright from Neuron, Cell Press. Panel B (right) is modified and reproduced with permission from Paxinos and Franklin (2001) The mouse brain in stereotaxic coordinates. Copyright from Academic Press.

of NMDA receptors may help shed further light on their involvement in experience-dependent plasticity (Bender et al., 2006; Clem et al., 2008) and the roles of presynaptic NMDA receptors (Brasier and Feldman, 2008). In future studies, it will be interesting to directly compare this Cre-loxP knockout approach with knockdown approaches based on siRNA (Mello and Conte, 2004). 


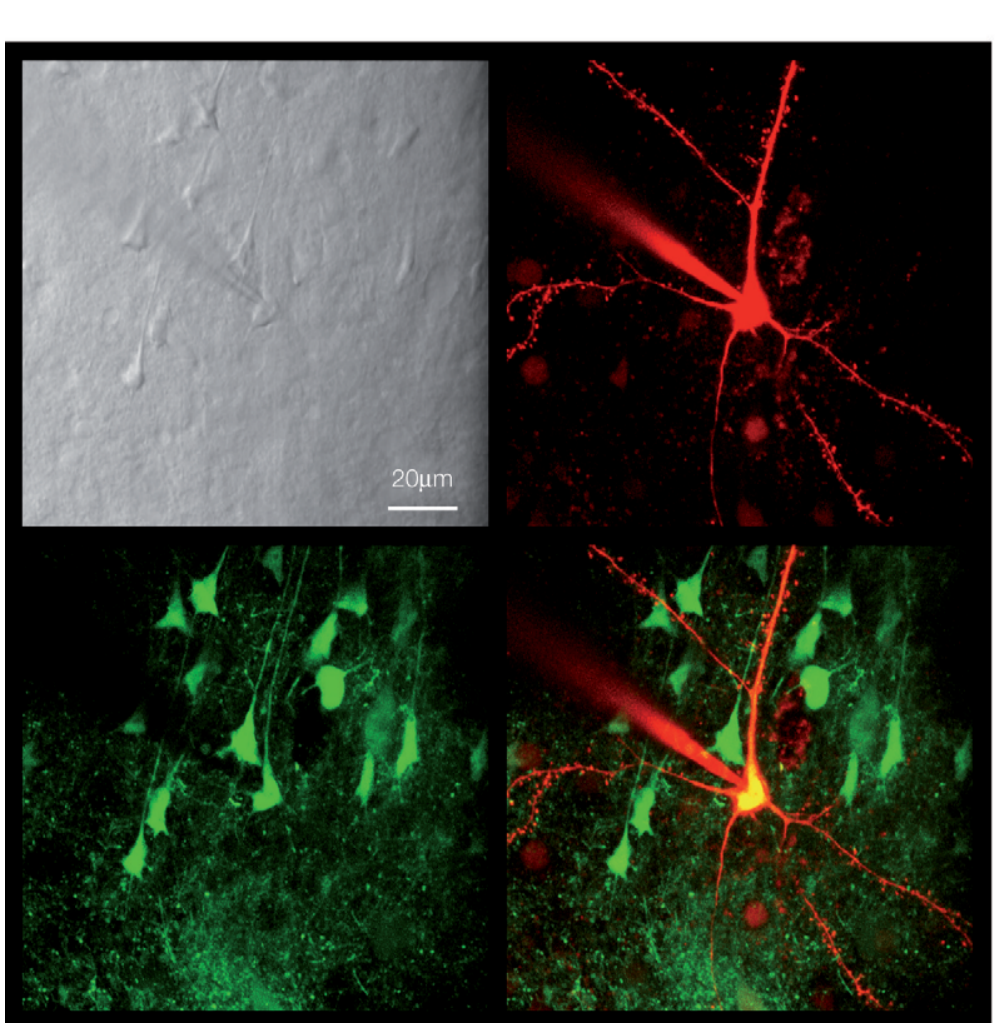

B

Control cell

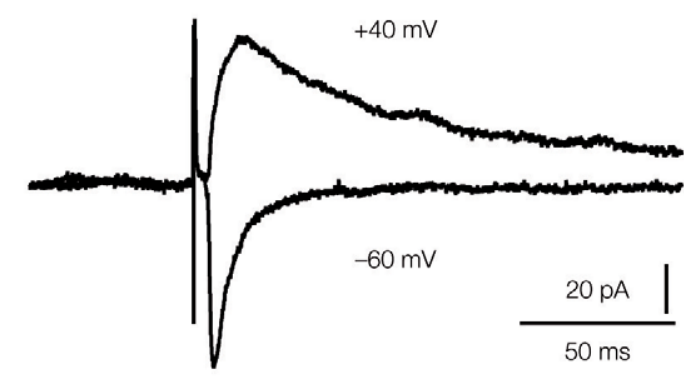

C NR1 knockout cell

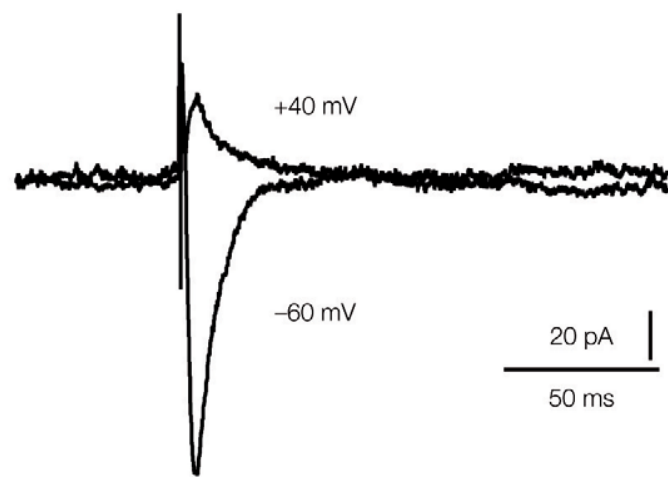

Figure 5 I Highly controlled knockout of NMDA receptors in the barrel cortex. (A) Imaging by two-photon microscopy of lentivector transduced pyramidal neurons expressing GFP and Cre recombinase in a floxed NR1 mouse. The Dodt contrast image (upper left), shows the whole-cell recording electrode filled with Alex 594 (upper right) targeted to a GFP expressing neuron (lower left, overlay lower right). (B) Whole-cell recording of a control layer 2/3 pyramidal neuron near the lentivector injection site. The neuron did not express GFP and had normal NMDA receptor dependent currents evoked by an extracellular stimulus in layer 4 and measured at $+40 \mathrm{mV}$. (C) Whole-cell recording of a layer 2/3 pyramidal neuron expressing GFP and Cre recombinase in a floxed NR1 mouse. Synaptically evoked currents lack the long-lasting NMDA-receptor dependent component at $+40 \mathrm{mV}$, indicating a functional knockout of NR1. Modified and reproduced with permission from Aronoff and Petersen (2007). Frontiers in Integrative Neuroscience.

The NMDA receptor provided an attractive first candidate for this analysis, since it is thought to play a pivotal role in learning and memory. The specific knockout of NMDA receptors in a single column and layer of the mouse somatosensory cortex might be of particular interest to combine with behaviours mediated by a single whisker. Using these strategies one could ask highly specific questions relating to the functional contributions of individual genes to specific behaviours. For example, one might examine whether the knockout of NMDA receptors in layer $2 / 3$ of the C2 barrel column would affect learning of the gap-crossing task with the single C2 whisker. There are increasing numbers of genes that have been floxed, and their functional roles in the mouse barrel cortex could be investigated with similar approaches. In the mouse barrel cortex such highly specific genetic manipulations can not only be correlated with learned behaviours, but also be investigated in terms of specific changes to welldefined synaptic circuits.

\section{FUTURE PERSPECTIVES}

The mouse barrel cortex offers a relatively simple sensory system amenable to detailed analysis. The anatomical map of the barrels allow simple alignment and comparison of data obtained from different animals. Both imaging and electrophysiological methods have been applied to study the functional activity of the somatosensory cortex of awake behaving mice (Crochet and Petersen, 2006; Dombeck et al., 2007; Ferezou et al., 2007). In addition to correlating neuronal activity with anatomical structure and behaviour, it is critical to obtain causal evidence for the involvement of specific neurons and to investigate the underlying mechanisms. Local genetic manipulations offered by viral gene transduction provide a simple route to first order layer, column and cell-type specificity. Genetically manipulated cells expressing GFP can be visualized at high resolution in vivo using two-photon microscopy (Dittgen et al., 2004; Stettler et al., 2006), but this is usually limited to the superficial cortical 
layers. Advances in endoscopic imaging technology may enable application of these methods for studying deep brain areas (Flusberg et al., 2005), but inevitably this will result in some damage to the brain upon the insertion of optical fibres.

In this review we have focused exclusively on the application of lentivector to mouse neocortex, but other viruses, such as adeno-associated virus, have been shown to provide equally stable vectors for gene delivery (recently reviewed Aronoff and Petersen, 2006; Luo et al., 2008). Lentivirus and adeno-associated virus have broadly similar characteristics in the context of expressing transgenes in the mammalian brain. Lentivirus can support slightly larger transgenes than adeno-associated virus, which has a smaller physical size and therefore also diffuses further from the injection site. Because the adeno-associated virus does not prominently integrate into the host cell genome, it therefore has the advantage of not disrupting native gene expression patterns, which might occur from a lentivector integration site. Importantly, both lentivirus and adeno-associated virus have been shown to work well in many brain areas of both rodents and primates (Aronoff and Petersen, 2006; Luo et al., 2008; Naldini et al., 1996; Stettler et al., 2006; Xiao et al., 1999), and indeed using viral vectors provides an important translational research direction towards gene therapy. Future developments in vector technology using combinatorial genetic methods will likely further increase the cell-type specificity (Wickersham et al., 2007a,b). Through iterative cycles of computational modelling, neurophysiology and genetic manipulation in mouse barrel cortex, it seems likely that we will ultimately gain a deep insight into basic cortical functions underlying sensory perception and associative learning.

\section{CONFLICT OF INTEREST STATEMENT}

The authors declare that the research was conducted in the absence of any commercial or financial relationships that could be construed as a potential conflict of interest.

\section{REFERENCES}

Accolla, R., Bathellier, B., Petersen, C. C. H., and Carleton, A. (2007). Differential spatial representation of taste modalities in the rat gustatory cortex. J. Neurosci. 27, 1396-1404.

Aronoff, R., and Petersen, C. C. H. (2006). Controlled and localized genetic manipulation in the brain. J. Cell. Mol. Med. 10, 333-352.

Aronoff, R., and Petersen, C. C. H. (2007). Layerand column-specific knockout of NMDA receptors in pyramidal neurons of the mouse barrel cortex. Front. Integr. Neurosci. 1, 1. doi: 10.3389/neuro.07.001.2007.

Bender, V. A., Bender, K. J., Brasier, D. J., and Feldman, D. E. (2006). Two coincidence detectors for spike timing-dependent plasticity in somatosensory cortex. J. Neurosci. 26, 4166-4177.

Binzegger, T., Douglas, R. J., and Martin, K. A. (2004). A quantitative map of the circuit of cat primary visual cortex. J. Neurosci. 24, 8441-8453.

Bonhoeffer, T., and Grinvald, A. (1991). Iso-orientation domains in cat visual cortex are arranged in pinwheel-like patterns. Nature 353, 429-431.

Brasier, D. J., and Feldman, D. E. (2008). Synapsespecific expression of functional presynaptic NMDA receptors in rat somatosensory cortex. J. Neurosci. 28, 2199-2211.

Brecht, M. (2007). Barrel cortex and whisker-mediated behaviors. Curr. Opin. Neurobiol. 17, 408-416.

Broser, P., Grinevich, V., Osten, P., Sakmann, B., and Wallace, D. J. (2007). Critical period plasticity of axonal arbors of layer $2 / 3$ pyramidal neurons in rat somatosensory cortex: layer-specific reduction of projections into deprived cortical columns. Cereb. Cortex. doi: 10.1093/cercor/bhm189.

Clem, R. L., Celikel, T., and Barth, A. L. (2008). Ongoing in vivo experience triggers synaptic metaplasticity in the neocortex. Science 319, 101-104.

Crochet, S., and Petersen, C. C. H. (2006). Correlating whisker behavior with membrane potential in barrel cortex of awake mice. Nat. Neurosci. 9, 608-610.

Deglon, N., Tseng, J. L., Bensadoun, J. C., Zurn, A. D., Arsenijevic, Y., Pereira de Almeida, L., Zufferey, R., Trono, D., and Aebischer, P. (2000). Self-inactivating lentiviral vectors with enhanced transgene expression as potential gene transfer system in Parkinson's disease. Hum. Gene Ther. 11, 179-190.

Dittgen, T., Nimmerjahn, A., Komai, S., Licznerski, P., Waters, J., Margrie, T. W., Helmchen, F., Denk, W., Brecht, M., and Osten, P. (2004). Lentivirus-based genetic manipulations of cortical neurons and their optical and electrophysiological monitoring in vivo. Proc. Natl. Acad. Sci. USA 101, 18206-18211.

Dombeck, D. A., Khabbaz, A. N., Collman, F., Adelman, T. L., and Tank, D. W. (2007). Imaging large-scale neural activity with cellular resolution in awake, mobile mice. Neuron 56, 43-57.

Ferezou, I., Bolea, S., and Petersen, C. C. H. (2006). Visualizing the cortical representation of whisker touch: voltage-sensitive dye imaging in freely moving mice. Neuron 50, 617-629.

Ferezou, I., Haiss, F., Gentet,L.J.,Aronoff, R., Weber, B., and Petersen, C. C. H. (2007). Spatiotemporal dynamics of cortical sensorimotor integration in behaving mice. Neuron 56, 907-923.

Flusberg, B. A., Cocker, E. D., Piyawattanametha, W., Jung, J. C., Cheung, E. L., and Schnitzer, M. J. (2005). Fiber-optic fluorescence imaging. Nat. Methods 2, 941-950.

Grinevich, V., Brecht, M., and Osten, P. (2005). Monosynaptic pathway from rat vibrissa motor cortex to facial motor neurons revealed by lentivirus-based axonal tracing. J. Neurosci. 25, 8250-8258.

Harris, J. A., Petersen, R. S., and Diamond, M. E. (1999). Distribution of tactile learning and its neural basis. Proc. Natl. Acad. Sci. USA 96, 7587-7591.
Hutson, K. A., and Masterton, R. B. (1986). The sensory contribution of a single vibrissa's cortical barrel. J. Neurophysiol. 56, 1196-1223.

Iwasato, T., Datwani, A., Wolf, A. M., Nishiyama, H., Taguchi,Y.,Tonegawa,S., Knopfel, T., Erzurumlu, R. S., and Itohara, S. (2000). Cortex-restricted disruption of NMDAR1 impairs neuronal patterns in the barrel cortex. Nature 406, 726-731.

Kaas, J. H. (1991). Plasticity of sensory and motor maps in adult animals. Annu. Rev. Neurosci. 14, 137-167.

Kalatsky, V. A., Polley, D. B., Merzenich, M. M., Schreiner, C. E., and Stryker, M. P. (2005). Fine functional organization of auditory cortex revealed by Fourier optical imaging. Proc. Natl. Acad. Sci. USA 102, 13325-13330.

Kleinfeld, D., Ahissar, E., and Diamond, M. E. (2006). Active sensation: insights from the rodent vibrissa sensorimotor system. Curr. Opin. Neurobiol. 16, 435-444.

Komai, S., Licznerski, P., Cetin, A., Waters, J., Denk, W., Brecht, M., and Osten, P. (2006). Postsynaptic excitability is necessary for strengthening of cortical sensory responses during experience-dependent development. Nat. Neurosci. 9, 1125-1133.

Krupa, D. J., Wiest, M. C., Shuler, M. G., Laubach, M., and Nicolelis, M. A. (2004). Layer-specific somatosensory cortical activation during active tactile discrimination. Science 304, 1989-1992.

Luo, L., Callaway, E. M., and Svoboda, K. (2008). Genetic dissection of neural circuits. Neuron 57, 634-660.

Mello, C. C., and Conte, D. (2004). Revealing the world of RNA interference. Nature 431, 338-342.

Naldini, L., Blomer, U., Gallay, P., Ory, D., Mulligan, R., Gage, F. H., Verma, I. M., and Trono, D. (1996). In vivo gene delivery and stable transduction of nondividing cells by a lentiviral vector. Science 272, 263-267. 
Paxinos, G., and Franklin, K. B. J. (2001). The Mouse Brain in Stereotaxic Coordinates, 2nd Edn. San Diego, CA, Academic Press.

Petersen, C. C. H. (2007). The functional organization of the barrel cortex. Neuron 56, 339-355.

Petersen, C. C. H., and Sakmann, B. (2001). Functionally independent columns of rat somatosensory barrel cortex revealed with voltage-sensitive dye imaging. J. Neurosci. 21, 8435-8446.

Sarid, L., Bruno, R., Sakmann, B., Segev, I., and Feldmeyer, D. (2007). Modeling a layer 4-to-layer 2/3 module of a single column in rat neocortex: interweaving in vitro and in vivo experimental observations. Proc. Natl. Acad. Sci. USA 104, 16353-16358.

Shepherd, G. M., Pologruto, T. A., and Svoboda, K. (2003). Circuit analysis of experience-dependent plasticity in the developing rat barrel cortex. Neuron 38, 277-289.

Silberberg, G., Grillner, S., LeBeau, F. E., Maex, R., and Markram, H. (2005) Synaptic pathways in neural microcircuits. Trends Neurosci. 28, 541-551.

Soriano, P. (1999). Generalized lacZ expression with the ROSA26 Cre reporter strain. Nat. Genet. 21, $70-71$
Stettler, D. D., Yamahachi, H., Li, W., Denk, W., and Gilbert, C. D. (2006). Axons and synaptic boutons are highly dynamic in adult visual cortex. Neuron $49,877-887$.

Thomson, A. M., and Lamy, C. (2007). Functional mapsof neocorticallocalcircuitry.Front.Neurosci. 1, 19-42. doi: 10.3389/neuro.01/1.1.002.2007

Tsien,J.Z.,Chen,D.F.,Gerber,D., Tom,C., Mercer, E. H., Anderson, D. J., Mayford, M., Kandel, E. R., and Tonegawa, S. (1996a). Subregion- and cell typerestricted gene knockout in mouse brain. Cell 87, 1317-1326.

Tsien, J. Z., Huerta, P. T., and Tonegawa, S. (1996b). The essential role of hippocampal CA1 NMDA receptor-dependent synaptic plasticity in spatial memory. Cell 87, 1327-1338.

Welker, E., Armstrong-James, M., Bronchti, G., Ourednik, W., Gheorghita-Baechler, F., Dubois, R., Guernsey, D. L., Van der Loos, H., and Neumann, P. E. (1996). Altered sensory processing in the somatosensory cortex of the mouse mutant barrelless. Science 271, 1864-1867.

Wickersham, I. R., Finke, S., Conzelmann, K. K., and Callaway, E. M. (2007a). Retrograde neuronal tracing with a deletion-mutant rabies virus. Nat. Methods 4, 47-49.
Wickersham, I. R., Lyon, D. C., Barnard, R. J., Mori, T., Finke, S., Conzelmann, K. K., Young, J. A., and Callaway, E.M. (2007b). Monosynaptic restriction of transsynaptic tracing from single, genetically targeted neurons. Neuron 53, 639-647.

Woolsey, T. A., and Van der Loos, H. (1970). The structural organization of layer IV in the somatosensory region (SI) of mouse cerebral cortex. The description of a cortical field composed of discrete cytoarchitectonic units. Brain Res. 17, 205-242.

Xiao, W., Chirmule, N., Berta, S. C., McCullough, B., Gao, G., and Wilson, J. M. (1999). Gene therapy vectors based on adeno-associated virus type $1 . J$ Virol. 73, 3994-4003.

Received: 26 March 2008; accepted: 02 May 2008.

Citation: Front. Neurosci. (2008) 2, 1: 64-71, doi: 10.3389/neuro.01.001.2008

Copyright () 2008 Aronoff and Petersen. This is an open-access article subject to an exclusive license agreement between the authors and the Frontiers Research Foundation, which permits unrestricted use, distribution, and reproduction in any medium, provided the original authors and source are credited. 